Figueiras, FN, Duarte, ML \& Santos, LR. (2020). Cerebellar pilocytic astrocytoma: literature review and case report of a young patient with anatomopathological correlation. Research, Society and Development, 9(7): 1-12, e405974115.

\title{
Astrocitoma pilocítico cerebelar: revisão da literatura e relato de caso de um jovem paciente com correlação anátomopatológica
}

Cerebellar pilocytic astrocytoma: literature review and case report of a young patient with anatomopathological correlation

Astrocitomas pilocíticos cerebelares: revisión de la literatura e informe de caso de un paciente joven con correlación anatomopatológica

Recebido: 30/04/2020 | Revisado: 02/05/2020 | Aceito: 10/05/2020 | Publicado: 20/05/2020

Felipe Nunes Figueiras

ORCID: https://orcid.org/0000-0001-6626-6731 Radiologista no Hospital Guilherme Álvaro, Santos, São Paulo, Brasil E-mail: bilita88@gmail.com Márcio Luís Duarte ORCID: https://orcid.org/ 0000-0002-7874-9332 WEBIMAGEM, São Paulo, São Paulo, Brasil. E-mail: marcioluisduarte@gmail.com Lucas Ribeiro dos Santos

ORCID: https://orcid.org/ 0000-0001-7897-1198 Faculdade de Ciências Médicas de Santos, Santos, são Paulo, Brasil E-mail: 1rs.endocrino@gmail.com

\section{Resumo}

O astrocitoma pilocítico (AP) é um tumor tipicamente circunscrito, de crescimento lento, sendo o glioma mais comum em crianças, geralmente originário do cerebelo; existem poucos relatos desse tumor em adultos, porém apresenta um bom prognóstico. $\mathrm{O}$ objetivo do presente artigo é relatar um diagnóstico AP em paciente adolescente cuja única queixa era cefaleia progressiva e pré-síncope. Dado que é fundamental o reconhecimento do padrão de imagem de um astrocitoma pilocítico pouco agressivo (grau 1) uma vez que pode se assemelhar a gliomas de alto grau, acarretando tratamentos mais agressivos equivocadamente, revisamos o papel dos métodos de imagem no diagnóstico do AP.

Palavras chave: Neoplasias de sistema nervoso central; Ressônancia magnética; Cerebelo. 


\begin{abstract}
Pilocytic astrocytoma (PA) is a typically circumscribed, slow-growing tumor, being the most common glioma in children, usually originating in the cerebellum; there are few reports of this tumor in adults, but it has a good prognosis. Here, we report an PA diagnosis in an adolescent patient whose only complaint was progressive headache and presyncope. Given that it is essential to recognize the image pattern of a little aggressive pilocytic astrocytoma (grade 1), as it can be similar to high-grade gliomas, leading to more aggressive treatments mistakenly, we review the role of imaging methods in the diagnosis of PA.
\end{abstract}

Keywords: Central nervous System neoplasms; Magnetic resonance; Cerebellum.

\title{
Resumen
}

El astrocitoma pilocítico (AP) es un tumor de crecimiento lento, típicamente circunscrito, que es el glioma más común en niños, que generalmente se origina en el cerebelo; Hay pocos informes de este tumor en adultos, pero tiene un buen pronóstico. Aquí, informamos un diagnóstico de AP en un paciente adolescente cuya única queja fue dolor de cabeza progresivo y presíncope. Dado que es esencial reconocer el patrón de imagen de un astrocitoma pilocítico poco agresivo (grado 1), ya que puede ser similar a los gliomas de alto grado, lo que conduce a tratamientos más agresivos por error, revisamos el papel de los métodos de imagen en el diagnóstico de AP.

Palabras clave: Neoplasias del sistema nervioso central; Resonancia magnética; Cerebelo.

\section{Introdução}

O astrocitoma pilocítico (AP) é um tumor tipicamente circunscrito, de crescimento lento, sendo o glioma mais comum em crianças (Murahashi 2012; Bell 2004; Kelly 2004), correspondendo a cerca de $2-6 \%$ de todos os tumores cerebrais primários e com uma incidência de 0.91/100,000 pessoas por ano (Haaga 2010, Rocha 2012, Ostrom 2018), no qual a maioria surge no cerebelo, cerca de $60 \%$ dos casos, tendo os hemisférios cerebelares maior incidência que o vérmis (Haaga 2010), sendo, em contraste, o menos comum nos adultos (Kelly 2004, Bell 2004, Murahashi 2012). Acomete, primordialmente, as duas primeiras décadas de vida (Kelly 2004, Burger 2000), com pico de incidência em pacientes entre 5 e 15 anos (Haaga 2010, Rocha 2012) sem apresentar predileção por sexo (Rocha 2004, Kelly 2004, Burger 2000), considerado grau I pela Organização Mundial de Saúde (Rocha 2012, Robbins 2004). 
Em adultos, porém, a incidência diminui para apenas 0,8\% dos tumores do SNC em pacientes com mais de 19 anos (Ostrom, 2018). Nesses casos, os sintomas da doença se desenvolvem durante a terceira década de vida, sendo o aparecimento numa idade mais avançada extremamente rara (Yoshida 2011), mas apresenta um prognóstico favorável (Bell 2004). No entanto, nesta faixa etária, o local mais comum são os hemisférios cerebrais (Kelly 2004)

Os locais mais frequentemente envolvidos são os gânglios da base, quiasma óptico e hipotálamo como principais estruturas supratentoriais (Murahashi 2012), tendo em menor escala os hemisférios e ventrículos cerebrais (Kelly 2004), e o cerebelo no aspecto infratentorial (Murahashi 2012; Rocha 2012), seguido pelo tronco encefálico (Rocha 2013) que apresenta crescimento como massa exofítica dorsal (Shaya 2004).

O AP apresenta quadro clínico variável, com os sintomas mais comuns sendo cefaléia, náuseas e vômitos, bem como crises epilépticas devido a hidrocefalia secundária à obstrução do quarto ventrículo (Haaga 2010), além de visão turva, diplopia e cervicalgia (Kelly 2004). Fraqueza e perda de equilíbrio também podem ser relatados (Haaga 2010), assim como papiledema, paralisia do sexto par craniano (nervo abducente) e nistagmo (Kelly 2004). Puberdade precoce central secundária a um astrocitoma cerebelar é extremamente rara, sendo mais comum quando o astrocitoma acomete a região suprasselar (Rocha 2012).

Outra possível complicação é hemorragia tumoral; ela é apenas esporadicamente relatada e resultam de prováveis anormalidades na vascularização frequentemente observada nestes tumores, ocorrendo com maior frequência do que se pensava (White 2006).

O AP apresenta bom prognóstico (Murahashi 2012), com taxa de sobrevida em 10 anos de 94\% (Rocha 2012, Kelly 2004). O aumento da taxa de sobrevivência em 10 anos (cerca de $90 \%$ a partir de 1970, em comparação com cerca de 70\% antes de 1970) tem sido atribuida a melhorias nas técnicas e equipamento neurocirúrgicos.

Metástases não necessariamente aumentam a mortalidade, em contraste com o mau prognóstico tão comum em pacientes com tumores de alto grau metastático (Kelly 2004). Disseminação a distância de um astrocitoma pilocítico é rara, com uma prevalência de $2 \%$ a $12 \%$ dos casos, tendendo a se manifestar em um prazo de 3 anos após o diagnóstico inicial (Kelly 2004).

Sua recorrência é imprevisível, bem como a falta de recursos para prever as raras alterações malignas após a primeira remoção que representam um problema desafiador (Kelly 2004). Já foi documentada recorrência do tumor 36 anos após a remoção inicial (Pagni 1991). O objetivo do presente artigo é relatar um diagnóstico AP em paciente adolescente cuja única 
(CC BY 4.0) | ISSN 2525-3409 | DOI: http://dx.doi.org/10.33448/rsd-v9i7.4115

queixa era cefaleia progressiva e pré-síncope. Relatamos um caso de um astrocitoma pilocítico em paciente jovem e revisamos a literatura a cerca do comportamento clínico dessa condição, bem como os achados em RM

\section{Metodologia}

Uma pesquisa é realizada com a finalidade de se trazer novos saberes para a sociedade como preconiza Pereira, Shitsuka, Parreira e Shitsuka (2018). No presente trabalho, realiza-se um estudo de caso qualitativo relacionado às Ciências da Saúde. Neste tipo de estudo procurase detalhar com profundidade o caso específico.

\section{Relato do Caso}

Paciente do sexo masculino, 17 anos de idade, caucasiano, estudante, natural e procedente de Santos (SP), refere cefaléia há seis meses, com sensação de queimação na região posterior da nuca irradiando para topografia occipital, sem outros sintomas. Além disso, relatava dois episódios de pré-síncope. O exame físico apresentava-se inocente, sem qualquer déficit focal, Sinal de Romberg e força motora nos quatro membros e diadococinesia normais, bem como não apresentava nistagmo.

Relata varicela na infância, assim como cirurgias de hérnia inguinal, hérnia umbilical e amidalectomia, negando outras patologias e etilismo, tabagismo ou uso de drogas ilícitas. Refere três internações prévias por bronquite aguda causa da qual faz uso de sintomáticos, sem fazer uso de medicações de uso contínuo. Apresenta um irmão com bronquite crônica e mãe com lúpus eritematoso sistêmico. Demais familiares saudáveis. Realizada tomografia computadorizada (TC) conforme a Figura A com. 
Research, Society and Development, v. 9, n. 7, e405974115, 2020

(CC BY 4.0) | ISSN 2525-3409 | DOI: http://dx.doi.org/10.33448/rsd-v9i7.4115

Figura A - Tomografia computadorizada na seção axial, sem contraste.
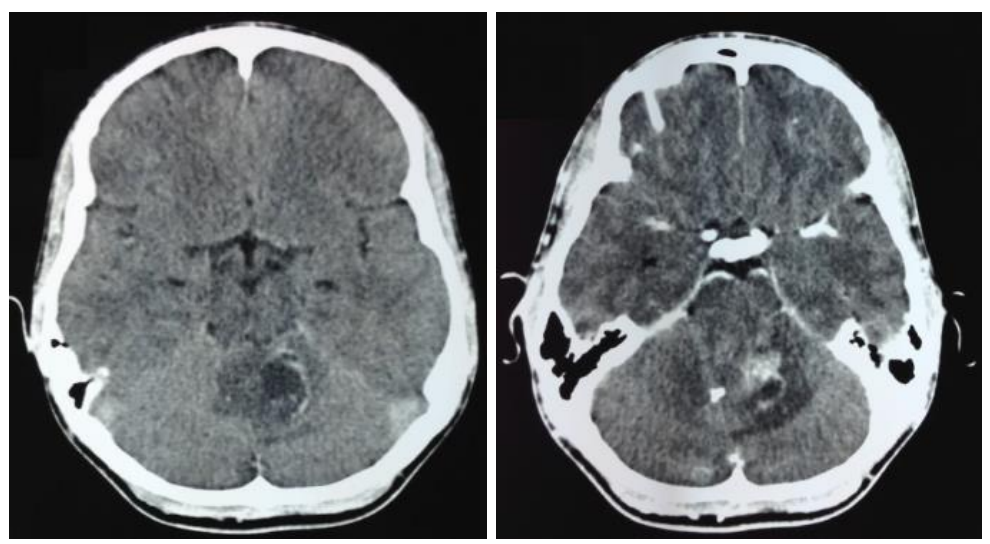

Fonte: Autores.

A seguir apresenta-se a Figura B da tomografia computadorizada realizada sem contraste.

Figura B - Tomografia computadorizada na seção axial, com contraste.

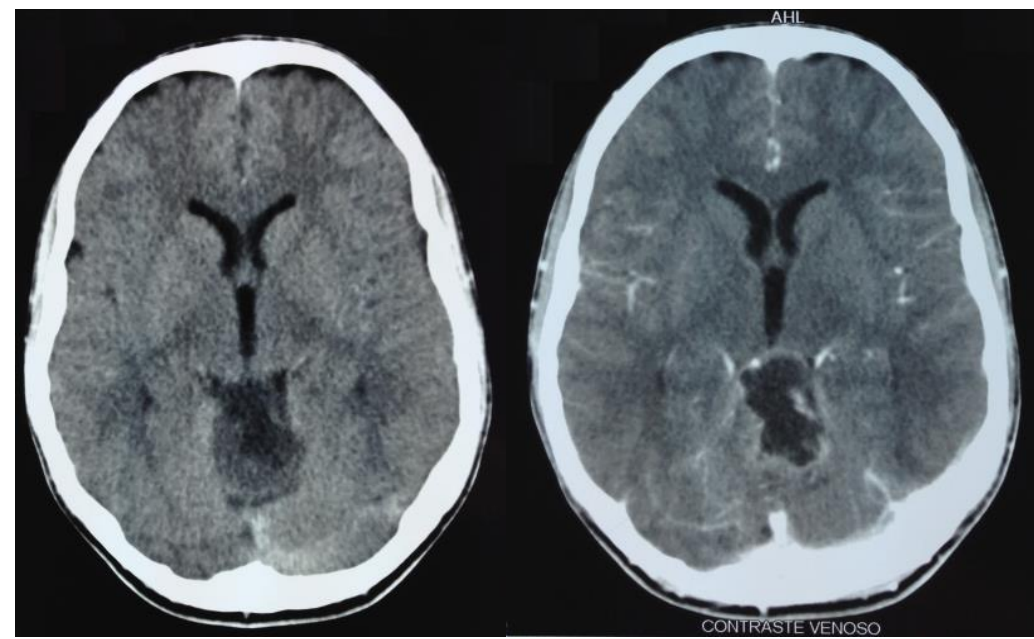

Fonte: Autores.

Constatou-se a formação expansiva, ovalada, heterogênea na projeção do hemisfério cerebelar superior médio-lateral esquerdo, com contornos irregulares e limites mal definidos, apresentando aspecto hiper e hipodenso, correspondendo à lesão sólido-cística, com realce periférico e de sua porção sólida após a infusão do meio de contraste iodado.

Observa-se edema periférico que promove efeito de massa sobre as estruturas adjacentes, com compressão e desvio caudal do quarto ventrículo com leve dilatação do sistema ventricular supratentorial, apresentando o ventrículo lateral direito de dimensões 
(CC BY 4.0) | ISSN 2525-3409 | DOI: http://dx.doi.org/10.33448/rsd-v9i7.4115

maiores, além de proliferação vascular serpiginosa perifericamente ao componente sólido.

A ressonância magnética ( $\mathrm{RM}$ - Figuras C, D e E) foi comprometida devido a presença de artefatos de suceptibilidade magnética relacionada a aparelho ortodôntico segundo dados clínicos - degradando as imagens particularmente das sequências Difusão e Gradiente-Echo. No entanto, foi possível destacar-se uma formação expansiva no vérmis cerebelar, estendendo-se a substância branca cerebelar esquerda, apresentando sinal hipointenso em T1 e hiperintenso e heterogêneo em T2 e FLAIR, com áreas císticas / necróticas intralesionais, notando-se também focos de marcante hipossinal na sequência T2 relacionados a produtos de degradação da hemoglobina ou mesmo calcificação, além de impregnação periférica, espessa e irregular pelo agente paramagnético. A lesão promove efeito de massa, deslocando o IV ventrículo caudal e anteriormente, promovendo consequente ectasia do sistema ventricular supratentorial. A Figura $\mathrm{C}$ apresenta a RC na seção axial sequência T1 sem contraste.

Figura C - Ressonância magnética na seção axial sequência T1 sem contraste.

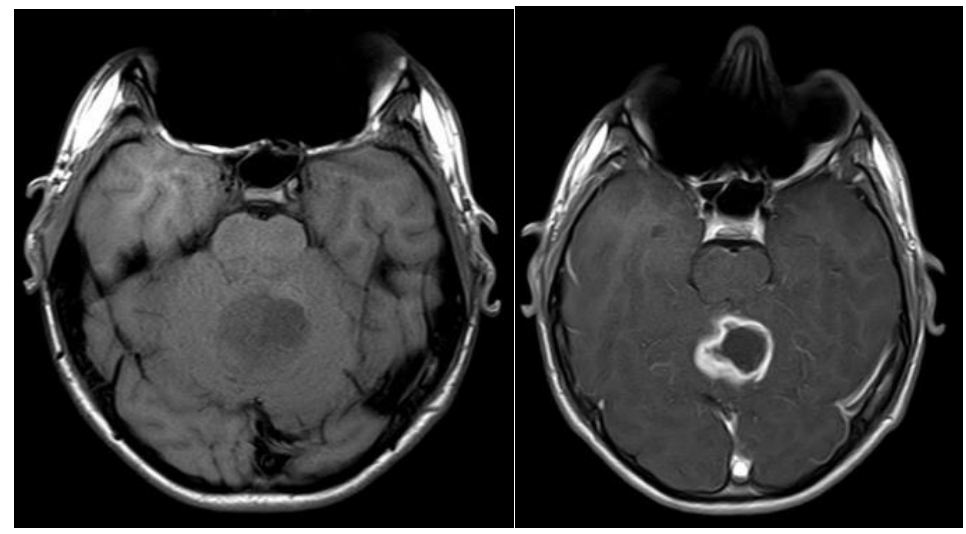

Fonte: Autores.

A Figura C apresenta imagens da RC na seção axial sequência T2 sem contraste. 
(CC BY 4.0) | ISSN 2525-3409 | DOI: http://dx.doi.org/10.33448/rsd-v9i7.4115

Figura D - Ressonância magnética na seção axial sequência T2 sem contraste.

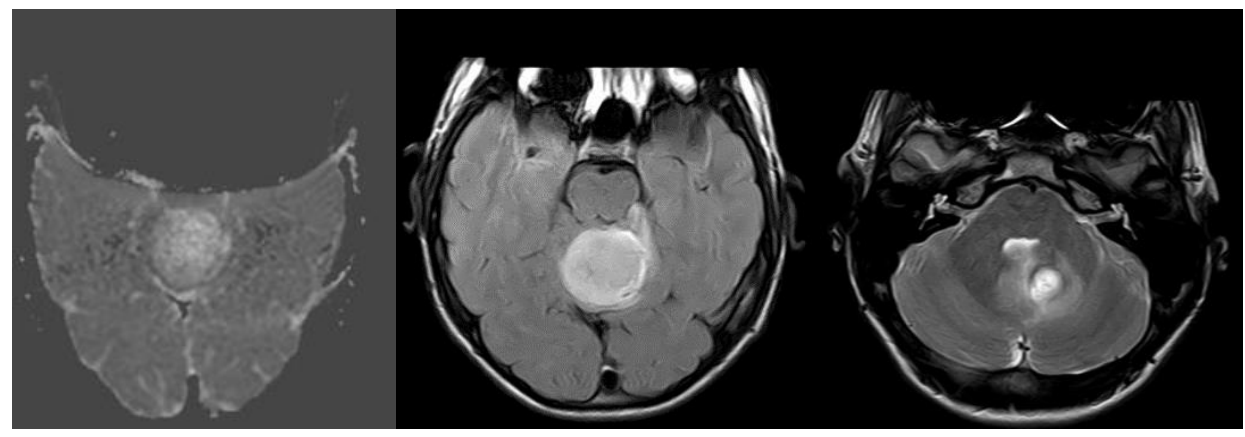

Fonte: Autores.

Já a Figura E ilustra a RC, sequência T1, com contraste em corte coronal e sagital.

Figura E - Ressonância magnética sequência T1 com contraste em corte coronal e sagital.

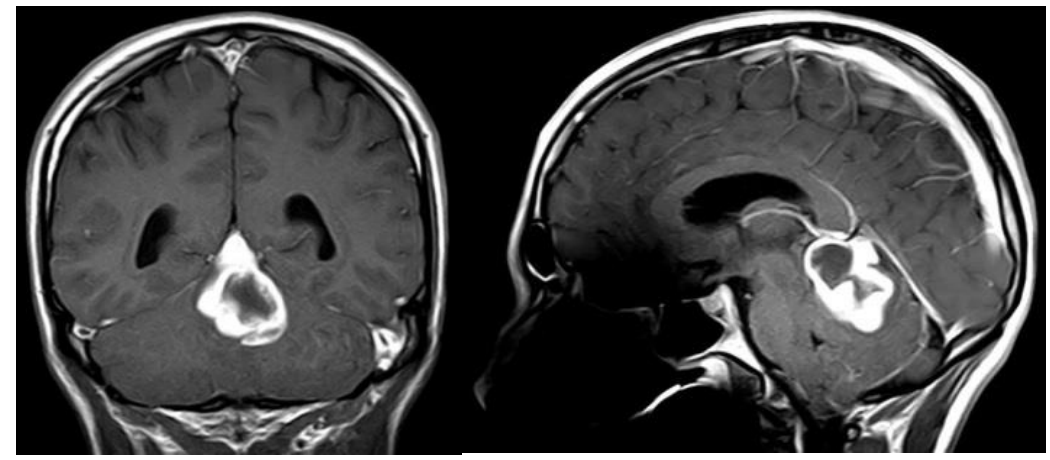

Fonte: Autores.

Realizada cirurgia com ressecção parcial da lesão e posterior tratamento radioterápico. A lesão, no intra-operatório apresentou contato com o mesencéfalo, não sendo, por isso, totalmente ressecada. $\mathrm{O}$ estudo anátomo-patológico e imuno-histoquímico que possibilitaram a conclusão de astrocitoma pilocítico cerebelar (Grau I, OMS).

Na tomografia computadorizada após a cirurgia que demonstrou lesão com aspecto heterogêneo associado a edema leve projetado no vérmis cerebelar com compressão do IV ventrículo. Contudo, o sistema ventricular supratentorial apresentava-se preservado.

\section{Discussão}

É importante reconhecer a aparência de imagem de um astrocitoma pilocítico pouco agressivo (grau 1), pois pode ser confundido com gliomas de alto grau, podendo levar a um 
tratamento equivocado.

Quatro padrões de imagens predominantes de astrocitoma pilocítico foram descritos (Kelly 2004), conforme constam no Quadro 1; nosso paciente em questão apresentava o padrão mais comum:

\begin{tabular}{|c|}
\hline Quadro 1: Características das imagens do astrocitoma pilocítico \\
\hline $\begin{array}{l}\text { Massa com um cisto que não realça ao contraste e um nódulo mural que realça intensamente } \\
\text { ( } 21 \% \text { dos casos). }\end{array}$ \\
\hline $\begin{array}{l}\text { Massa com uma parede cística que realça ao contraste e um nódulo mural que realça } \\
\text { intensamente ( } 46 \% \text { dos casos). }\end{array}$ \\
\hline Massa necrótica com uma zona central que não realça ao contraste (16\% dos casos). \\
\hline Massa predominantemente sólida com discreto componente cístico (17\% dos casos). \\
\hline
\end{tabular}

Fonte: Autores.

O aspecto tomográfico consiste em uma massa semelhante a um cisto hipodenso - de alto conteúdo proteico - e de margens regulares com um nódulo tumoral menor hipodenso e bem definido em uma das paredes com intenso realce homogêneo do nódulo após a infusão do contraste iodado - quando há realce das paredes do cisto, sugere-se um tumor mais agressivo (Haaga 2010). Pode ser completamente sólido em até $20 \%$ dos casos (Rocha 2012) e conter calcificações ocasionais (Kelly 2004).

Na RM o nódulo mural aparece homogeneamente hiperintenso em relação à substância cinzenta nas imagens ponderadas em T2 / FLAIR e hipo a isointenso em T1, sendo característica a intensificação homogênea deste nódulo após a infusão do gadolíneo (Haaha 2010, Rocha 2012), sendo, este, o melhor método para a avaliação do tumor (Rocha 2012). Focos calcificados podem dar um aspecto heterogêneo à lesão (Haaga 2010), além de não apresentar comumente edema perilesional (Haaga 2010, Rocha 2012) e não apresenta restrição à difusão da água (Rocha 2012).

Relatórios da espectroscopia da RM realizada nas porções de tecido mole de astrocitomas pilocíticos documentaram elevação da colina em relação ao N-acetilaspartato (NAA), com proporções que variam entre 1,80-3,40 (normal do cerebelo: 0,53-0,75) (Sutton 1992). Não há correlação entre a celularidade e a sequência de ADC nos tumores do cerebelo pediátricos comuns, embora a sequência de ADC seja útil no diagnóstico pré-operatório de tumores pediátricos cerebelares comuns e seu uso seja geralmente atribuído a diferenças na 
celularidade dos tumores, a celularidade tumoral não pode ser o único fator determinante das diferenças de difusividade (Korgün 2013).

O acompanhamento com exames de imagem, principalmente com a RM que é o principal método diagnóstico para esta neoplasia, dos pacientes com astrocitoma pilocítico cerebelar é recomendada a cada 3 meses para os dois primeiros anos, seguido de cada 6 meses por outros dois anos, e depois anualmente (Schneider 1992).

O estudo histológico se caracteriza pela presença das várias fibras de Rosenthal (Burger 2002) eosinofílicas no citoplasma das células tumorais, com raras mitoses e raras áreas de necrose (Haaga 2010, Robins 2004), além de aumento no número de vasos sanguíneos, normalmente com paredes espessadas ou com proliferação celular vascular (Robbins 2004). Podem apresentam calcificações intratumorais (5-25\% dos casos), mas hemorragia no tumor ou adjacente a ele é raro (Haaga 2010, Rocha 2012). Para classificação dos graus de agressividade de um tumor de sistema nervoso central, a Organização Mundial de Saúde (OMS) utiliza critérios baseados em atipia nuclear, mitoses, proliferação endotelial e necrose (Louis, 2007), sendo o Grau 1 aqueles que não apresentam nenhum desses critérios

Algumas avaliações genéticas também podem ser utilizadas para avaliar o risco desses tumores; os AP geralmente apresentam fusão do BRAF, mas ocasionalmente podem apresentar uma mutação BRAF V600E, fusão RAF1 ou duplicação intragênica de FGFR1 (Antonelli, 2018). Além disso, biomarcadores epigenéticos, especialmente a metilação do DNA e o índice de proliferação Ki-67 têm sido estudados com o intuito de fornecer informações sobre o status patológico, bem como auxiliar no prognóstico (Antonelli, 2018).

Muito raramente um astrocitoma pilocítico pode sofrer transformação maligna para uma aparência histológica agressiva. Nestes casos, tal tumor é chamado de astrocitoma pilocítico anaplásico (maligno). Embora tenham sido notificados casos fatais, como afirmam Kuroiwa (1999) e, Dirks 1994, este evento não necessariamente carrega um resultado menos favorável.

\section{Considerações Finais}

O estudo anátomo-patológico desempenha um papel importante na determinação da agressividade e da terapêutica, principalmente no que diz respeito à cirurgia. $\mathrm{O}$ tratamento ideal para um astrocitoma pilocítico cerebelar não consiste apenas na cirurgia com o objetivo de remoção total do tumor e radioterapia a fim de evitar a disseminação de células tumorais e metástases posteriores, mas também em um pós-tratamento com acompanhamento se 
baseando em exames de imagens pós-operatórios, de preferência a RM, que é o método de escolha para o estudo deste tumor, sendo este o método diagnóstico essencial para a terapêutica apropriada.

\section{Referências}

Antonelli M, Fadda A, Loi E, Moi L, Zavattari C, Sulas P, Gentilini D, Cameli C, Bacchelli E, Badiali M, Arcella A, Morra I, Giangaspero F \& Zavattari P. (2018). Integrated DNA methylation analysis identifies topographical and tumoral biomarkers in pilocytic astrocytomas. Oncotarget. Feb 12;9(17):13807-13821. DOI: 10.18632/oncotarget.24480.

Bell D, Chitnavis BP, Al-Sarraj S, Connor S, Sharr MM \& Gullan RW. Pilocytic astrocytoma of the adult--clinical features, radiological features and management. Br J Neurosurg. 2004 Dec;18(6):613-6.

Burger PC \& Scheithauer BW. The brain: tumors. In: Burger P, Scheithauer B, eds. Surgical pathology of the nervous system and its coverings. 4th ed. Philadelphia, Pa: Churchill Livingstone, 2002; 203-215.

Burger PC, Scheithauer BW, Paulus W, Szymas J, Giannini C \& Kleihues P. Pilocytic astrocytoma. In: Kleihues $\mathrm{P}$, Cavenee $\mathrm{W}$, eds. Pathology and genetics of tumours of the nervous system. Lyon, France: IARC, 2000; 45-51.

Dirks PB, Jay V, Becker LE, et al. Development of anaplastic changes in low-grade astrocytomas of childhood. Neurosurgery 1994; 34:68-78.

Haaga, JR. TC \& RM: Uma Abordagem Do Corpo Humano Completo. $5^{\text {a }}$ Edição [H. Lederman, trad.]. Clevland. Haaga. 2010.

Kelly K. Koeller, Elisabeth J. Rushing. From the Archives of the AFIP Pilocytic Astrocytoma: Radiologic-Pathologic Correlation1. RadioGraphics, 2004, Vol.24: 1693-1708. $10.1148 /$ rg. 246045146 . 
(CC BY 4.0) | ISSN 2525-3409 | DOI: http://dx.doi.org/10.33448/rsd-v9i7.4115

Korgün Koral, Derek Mathis, Barjor Gimi, Lynn Gargan, Bradley Weprin, Daniel C. Bowers, Linda Margraf. Common Pediatric Cerebellar Tumors: Correlation between Cell Densities and Apparent Diffusion Coefficient Metrics. Radiology, 2013, Vol.268: 532-537, 10.1148/radiol.13121362.

Kuroiwa T, Ohta T, Tsutsumi A. Malignant pilocytic astrocytoma in the medulla oblongata: case report. Brain Tumor Pathol 1999; 16:81-85.

Louis DN, Ohgaki H, Wiestler OD, Cavenee WK, Burger PC, Jouvet A, Scheithauer BW \& Kleihues P. The 2007 WHO classification of tumours of the central nervous system. Acta Neuropathol. 2007 Aug;114(2):97-109.

Murahashi T, Sato K, Ito T, Ozaki Y, Sugio H, Nakamura H \& Tanaka S. A case of pilocytic astrocytoma in an adult. No Shinkei Geka. 2012 Sep;40(9):793-7.

Ostrom QT, Gittleman H, Truitt G, Boscia A, Kruchko C \& Barnholtz-Sloan JS. CBTRUS Statistical Report: Primary Brain and Other Central Nervous System Tumors Diagnosed in the United States in 2011-2015. Neuro Oncol. 2018 Oct 1;20(suppl_4):iv1-iv86.

Pagni CA, Giordana MT \& Canavero S. Benign recurrence of a pilocytic cerebellar astrocytoma 36 years after radical removal: case report.Neurosurgery 1991; 28:606-609.

Robbins, SL. Patologia - Bases Patológicas das Doenças. $7^{\text {a }}$ Edição [JL. Santos, trad.]. Chicago. 2004.

Pereira, AS, Shitsuka, DM, Parreira, FJ \& Shitsuka, R. (2018). Metodologia da pesquisa científica. [e-book]. Santa Maria. Ed. UAB/NTE/UFSM. Disponível em: https://repositorio.ufsm.br/bitstream/handle/1/15824/Lic_Computacao_MetodologiaPesquisa-Cientifica.pdf? sequence $=1$.

Rocha, AJ. Série Colégio Brasileiro de Radiologia e Diagnóstico por Imagem: Encéfalo. São Paulo. CBR Encéfalo. 2012. 
Shaya MR, Fowler MR, Nanda A. Pilocytic astrocytoma presenting as an intrinsic brainstem tumor: case report and review of the literature. J La State Med Soc. 2004 Jan-Feb;156(1):336.

Schneider JH, Jr, Raffel C, McComb JG. Benign cerebellar astrocytomas of childhood. Neurosurgery 1992; 30:58-63.

Sutton LN, Wang Z \& Gusnard D, et al. Proton magnetic resonance spectroscopy of pediatric brain tumors. Neurosurgery 1992; 31:195-202.

White JB, Piepgras DG, Scheithauer BW \& Parisi JE. Rate of spontaneous hemorrhage in histologically proven cases of pilocytic astrocytoma. J Neurosurg. 2008 Feb;108(2):223-6.

Yoshida Y, Tsukada T, Hashimoto M \& Hayashi Y. Pilocytic astrocytoma of the cerebrum presenting in an elderly patient: a case report. No Shinkei Geka. 2011 Sep;39(9):865-9.

\section{Porcentagem de contribuição de cada autor no manuscrito}

$$
\begin{gathered}
\text { Felipe Nunes Figueiras }-40 \% \\
\text { Márcio Luís Duarte }-30 \% \\
\text { Lucas Ribeiro dos Santos }-30 \%
\end{gathered}
$$

